\title{
Integration and qualification of the Mini-EUSO telescope on board the ISS
}

\author{
G. Cambié, ${ }^{a, e, *}$ A. Belov,,${ }^{b, c}$ F. Capel, ${ }^{d}$ M. Casolino,,${ }^{a, e, f}$ A. Franceschi, ${ }^{g}$ P. Klimov, ${ }^{c}$ L. \\ Marcelli, ${ }^{a}$ T. Napolitano, ${ }^{f}$ P. Picozza, ${ }^{a}, e$ L.W. Piotrowski, ${ }^{f}$ E. Reali ${ }^{a}$ and M. Ricci ${ }^{g}$ \\ ${ }^{a}$ Università degli studi di Roma Tor Vergata, Dipartimento di Fisica \\ Roma, Italy \\ ${ }^{b}$ Faculty of Physics, M.V. Lomonosov Moscow State University \\ Moscow, Russia \\ ${ }^{c}$ Skobeltsyn Institute of Nuclear Physics, Lomonosov Moscow State University \\ Moscow, Russia \\ ${ }^{d}$ KTH Royal Institute of Technology \\ Stockholm, Sweden \\ e INFN, Sezione di Roma Tor Vergata \\ Roma, Italy \\ ${ }^{f}$ Riken \\ Wako, Japan \\ ${ }^{g}$ INFN-LNF \\ Frascati, Italy \\ E-mail: giorgio.cambie@roma2.infn.it
}

Mini-EUSO is a compact telescope $\left(37 \times 37 \times 62 \mathrm{~cm}^{3}\right)$ currently hosted on board the International Space Station. Mini-EUSO is devoted primarily to study Ultra High Energy Cosmic Rays (UHECR) above $10^{21} \mathrm{eV}$ but also to search for Strange Quark Matter (SQM), to observe Transient Luminous Event (TLE) in upper atmosphere, meteoroids, sea bioluminescence and space debris tracking. Mini-EUSO consist of a main optical system, the Photo Detector Module (PDM), sensitive to UV spectrum $(300 \div 400 \mathrm{~nm})$ and several ancillary sensors comprising a visible $(400 \div 780 \mathrm{~nm})$ and NIR $(1500 \div 1600 \mathrm{~nm})$ cameras and a $8 \times 8$ channels Multi-Pixel Photon Counter Silicon PhotoMultiplier (MPPC SiPM) array which will increase the Tecnological Readyness Level of this ultrafast imaging sensor. Mini-EUSO belongs to a novel set of missions committed to evaluate, for the first time, the capability of observing Cosmic Rays from a space-based The instrumentation, space-qualified tests will be shown.

$37^{\text {th }}$ International Cosmic Ray Conference (ICRC 2021)

July 12th - 23rd, 2021

Online - Berlin, Germany

\footnotetext{
*Presenter
} 


\section{Introduction}

Mini-EUSO [1] is a mission which belongs to the JEM-EUSO (Joint Experiment Missions - Extreme Universe Space Observatory) collaboration, which aim to study Ultra High Energy Cosmic Rays (UHECRs) from space for the first time. The collaboration has developed different former missions, which togheter with Mini-EUSO, are turning out to be a good test bench for the future, larger and better performing satellite, K-EUSO [2] (KLYPVE) and POEMMA [3] (Probe Of Extreme MultiMessenger Astrophysics). Mini-EUSO was launched on August the 22nd 2019 with the Soyuz MS-14 spacecraft (an unmanned cargo expedition) from the Bajkonur Cosmodrome (Kazakhstan) and now is accommodated on the Russian Zvezda module, facing a UV-transparent window in Nadir mode. Mini-EUSO is observing UV emissions looking at the Earth surface in the latitude range covered by the ISS $\left( \pm 51.6^{\circ}\right)$ This approach is unique, not only for the huge area covered, respect to the onground experiments, but also because both Earth's hemispheres can be observed with one instrument, thus applying the same systematic error.

\section{The telescope}

The main detector has a super-wide-field of view $\left(44^{\circ}\right)$ which allows to map an Earth ground area of $263 \times 263 \mathrm{~km}^{2}$ thanks to the optics which comprises two double sided, $25 \mathrm{~cm}$ diameter, PMMA Fresnel lenses which focuses light onto a 36 Hamamatsu Multi-Anode PhotoMultiplier Tubes (MAPMT), each of 64 channels for a total of 2304 pixels with spatial resolution of $0.8^{\circ}$ per pixel in timescales up to $2.5 \mu \mathrm{s}$ called GTU (Gate Time Unit). Each MAPMT is powered by a Cockroft-Walton power supply board which convert $28 \mathrm{~V}$ up to $1100 \mathrm{~V}$ and present a BG3 UV filter on the entry window. The front end electronics consist of 6 SPACIROC3 [4] (Spatial Photomultiplier Array Counting Integrated ReadOutChip) boards and a Xilinx Zynq XC7Z030 SoC board [5]. In addition to the main detector, Mini-EUSO contains a Firefly MV and a Chameleon Point Grey compact cameras for complementary measurements in the near infrared and visible range, three single pixel UV sensors used as switches for day/night transition and a 64 channels Multi-Pixel Photon Counter (MPPC) imaging SiPM C13365 module provided by Hamamatsu Photonics. The front end electronic for SiPMs read out consist of a multiplexing board and an additional Atmel 2560 microcontroller board. The housekeeping of all instruments and the data storage into USB flash drive, is managed by a PCIe/104 form factor CPU. The instrumentation is powered by the Low Voltage Power Supply and a custom power board. The LVPS consisting of three PCB modules mounting different Vicor DC-DC converter which stabilize the $28 \mathrm{~V}$ input voltage coming from ISS and provides power for all subsystems, preserving electronics from spike and polarization inversion. Primary and secondary grounds are galvanically isolated. The Mini-EUSO power consumption is around 55 W. In Fig. 1 the telescope hardware and integration step.

\section{Space Qualification}

The General Technical Requirements for Experiment, Equipment and Technical Documents on board ISS required several tests to be performed on the instrument: Electro-Magnetic Interference and Conductive (EMC/EMI); vibration and shock; high/low pressure, thermal and humidity functional tests, which are described in the following. 

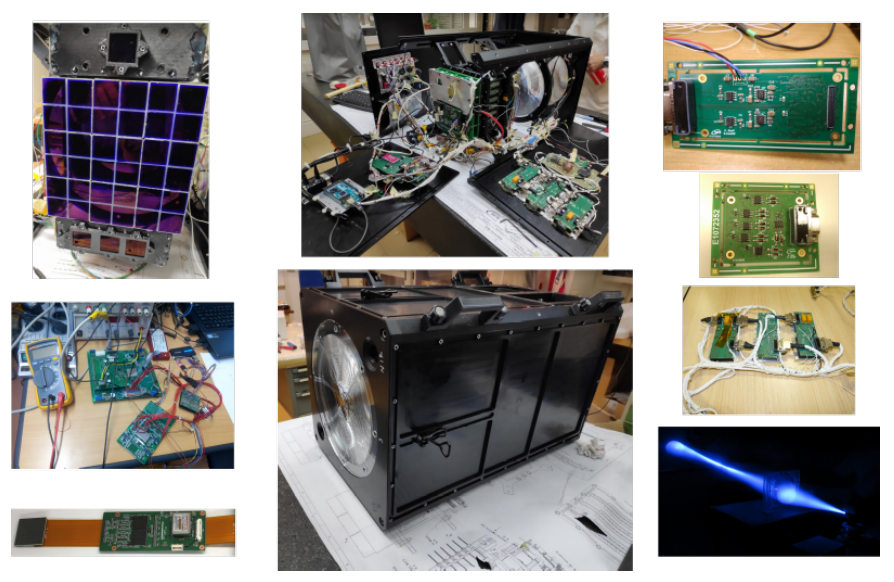

Figure 1: Mini-EUSO hardware and integration.

\subsection{EMC/EMI}

The aim of EMC/EMI tests is to verify that Mini-EUSO instrument does not produce any undesired electromagnetic radiated emissions and is capable to withstand different irradiations from external sources. In table 1all EMI/EMC qualifying tests.

Table 1: EMI/EMC tests specifications.

\section{EMI/EMC}

Low-Frequency (LF) Conductivve Emissions

High-Frequency (HF) Conductive Emissions

Electric Field Produced by HF Emissions

Low-Frequency Conductive Interference

Conductive HF Interference

$\begin{array}{ll}\text { Frequency range }(\mathrm{KHz}) & \text { Upeak }(d B \mu V) \\ 0.03 \div 10 & 100 \div 105 \\ 0.009 \div 100 & 60 \div 90 \\ 0.01 \div 1000 & 36 \div 60 \\ 0.02 \div 10 & 0.8 \div 1 \\ 100 \div 3 \times 10^{5} & 91 \div 120\end{array}$

Mini-EUSO must not generate interference levels greater than the thresholds specified when powered on both positive and negative wires at any supply voltages: $23,28,29 \mathrm{~V}$. The detector was arranged in the anechoic chamber in vertical, in a way such that the front end of the telescope faces the ground plane, with the front lens side touching the table. This is due to the fact that during operations the telescope faces to the ISS window. Compliance with the thresholds has been assured for both horizontally and vertically polarized waves using different types of antennas (Fig. 2). Generators were used to inject a voltage ripple profile into the input power lead and pulses with different amplitudes and durations. o comply with the specifications for most of the circuit protection elements used in the onboard hardware control system also Inrush current tests were done. Start-up currents shall not exceed five times the maximum steady-state operating current during power input voltage changes and pulses rise or fall rate should not exceed $0.125 \mathrm{~A} / \mu \mathrm{s}$ with a maximum energy of $0.1 A^{2} / s$. Mini-EUSO succesfully passed all EMC/EMI tests and the experience allowed the collaboration to improve electronics performances. In fig. 2 are shown the detector frequency profile responses, respectevely, when an external electric field is applied (left) and the high frequencies 
one conducted in normal operation (right).
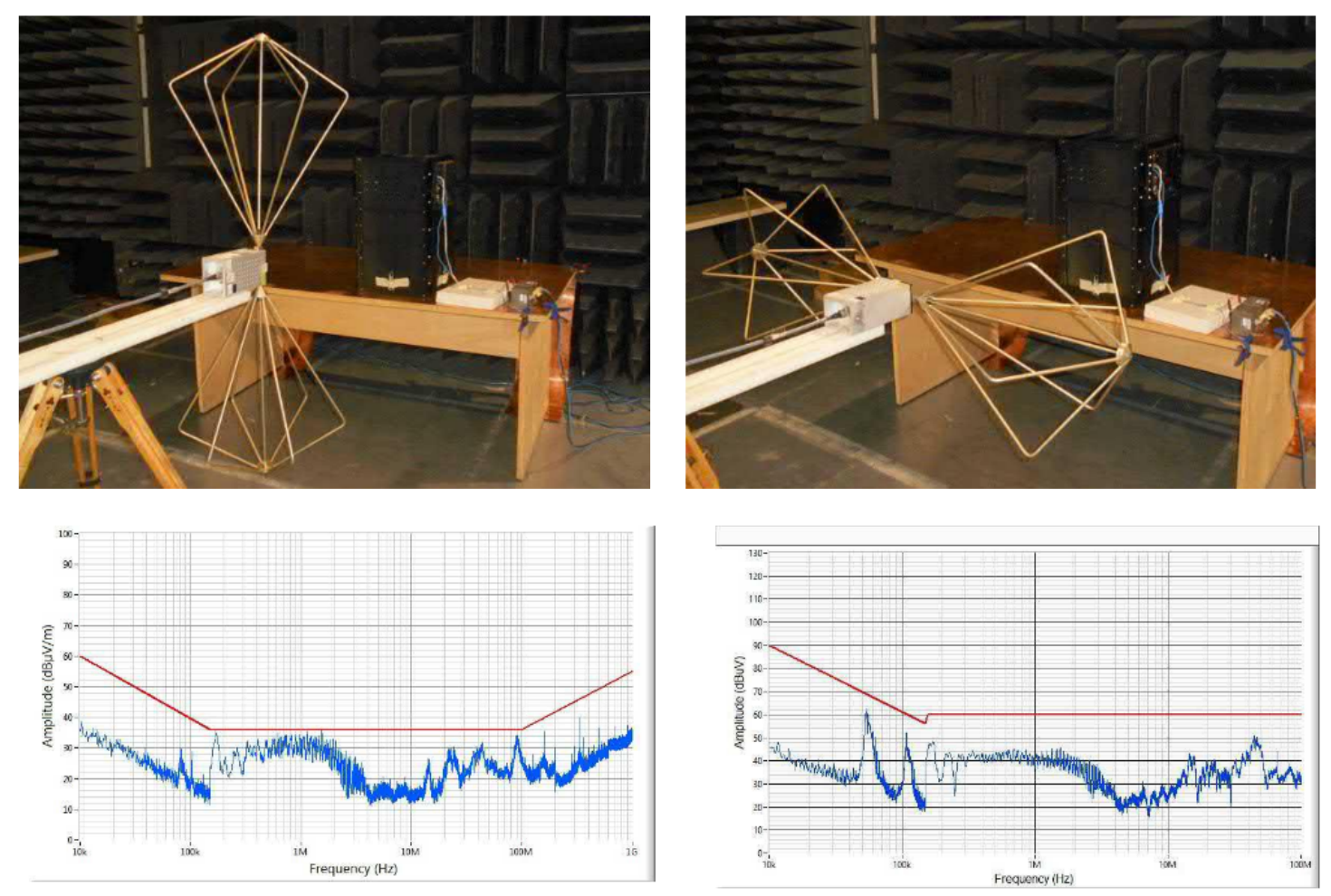

Figure 2: Top: Mini-EUSO Electric Field Interference tests (pol. V and H.). Bottom Left: E field Interference results. Bottom Right: HF conductive emissions results.

\subsection{Temperature, Pressure and Humidity}

Pressure test can be divided into low and high pressure tests, each of them performed with pressure, temperature and humidity sensors read by a Raspberry Py Single Board Computer. Those requires the detector to be left inside a special chamber for two hours reaching pressures of 450 and 760 $\mathrm{mm} \mathrm{Hg}$ in steps of $10 \mathrm{~mm} \mathrm{Hg}$ per second. After reaching standard atmosphere Mini-EUSO normal functionality has to be checked. Temperature test requires the detector to be installed into a thermal chamber for a total of 6 hours in which it was kept respectevely at $\pm 53^{\circ} \mathrm{C}$. Check of nominal functionality was done after reaching again standard temperature. The thermal cycles is shown in Fig. 3, while the pressure one is visible in Fig. 4. Mini-EUSO electronics and optics have not been damaged at all and this is a milestone for future detector manufacturing.

\subsection{Vibration and Shock}

Qualification vibration test requires random vibration and shock. Tri-axial accelerometer for monitoring were located on the HW, while a mono-axial one was fixed to the vibrating plate as feedback. A Resonance Survey was applied before and after each of the spectrums, where its level 
High Temperature second phase
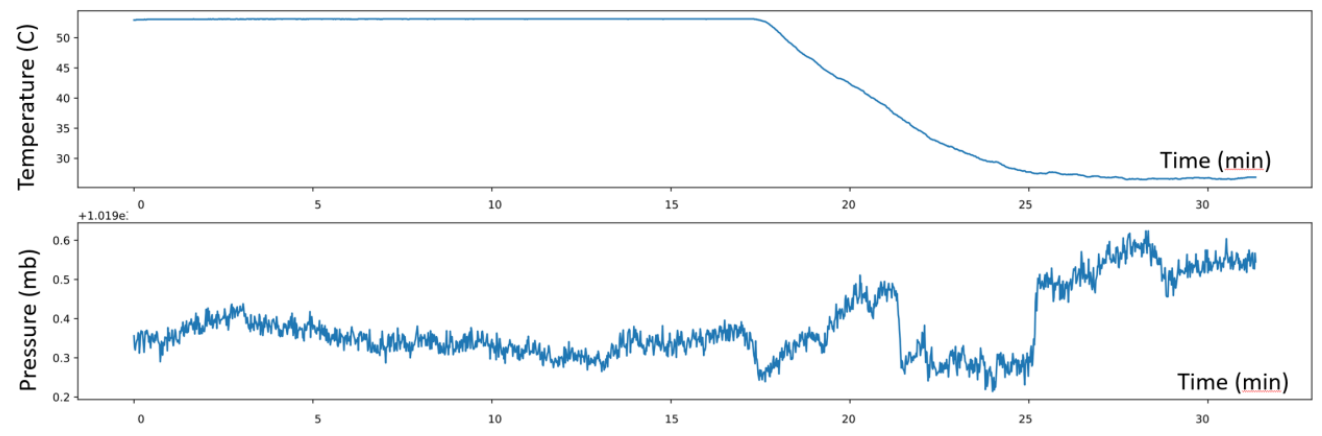

Figure 3: High temperature cycle.

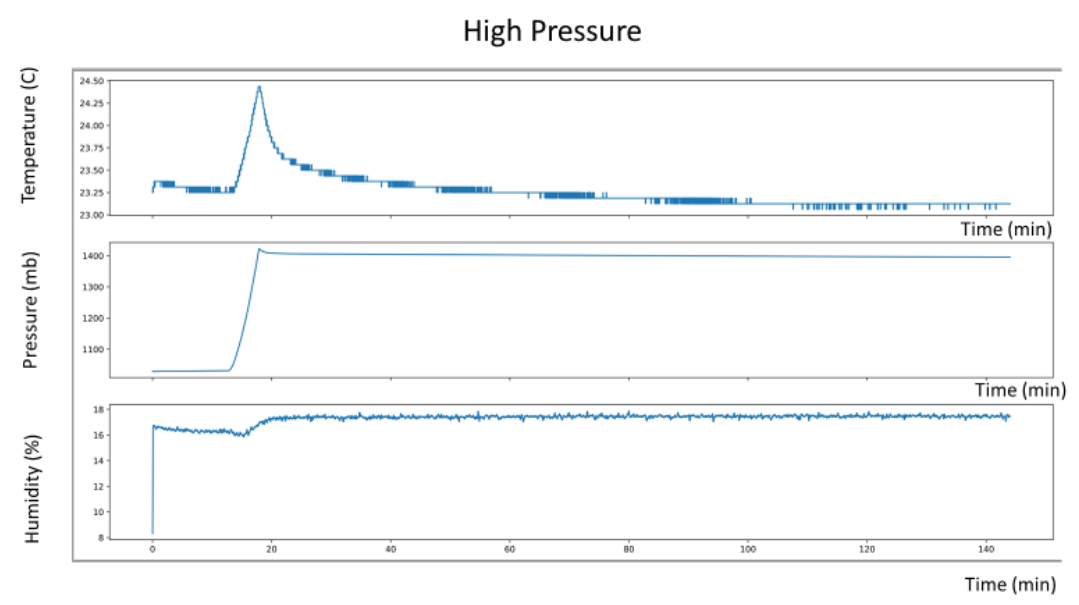

Figure 4: High pressure cycle.

is defined as 0.5 sine - 2octave/min - 1 sweepuponly $(20 \div 2000 \mathrm{~Hz})$. Random insertions were applied for 120, 480 and 600 seconds. The shock loads have a short time effect but with a high acceleration value $( \pm 40 \mathrm{G})$. The two curves, before and after the tests, were compared. Each test is considered successfully completed if, after the visual inspection, the equipment under test has maintained its physical characteristics, even if resonance discrepancies exceed for more than $5 \%$. The detector resonances exceeded the thresolds in some tests even if no structural breaks has been exhibited and normal operations were succesful. In Fig. 5 are shown plots of the resonance response after vibration and shock.

\section{Calibration}

Mini-EUSO is intended to work in a single photoelectron (PE) counting mode which has advantages over analog measurement in terms of signal-to-noise ratio. The 64 signals from MAPMT anodes are digitalized and discriminated to count photon triggered pulses and to measure the photon intensity, thus, allowing to set a threshold for the MAPMT single PE detection. This analysis is made through the S-curve plot which showes the number of triggered pulses as a function of the ADC pulse height threshold. Each channels is an 8 bit threshold step, so that we can distinguish the typical noise 

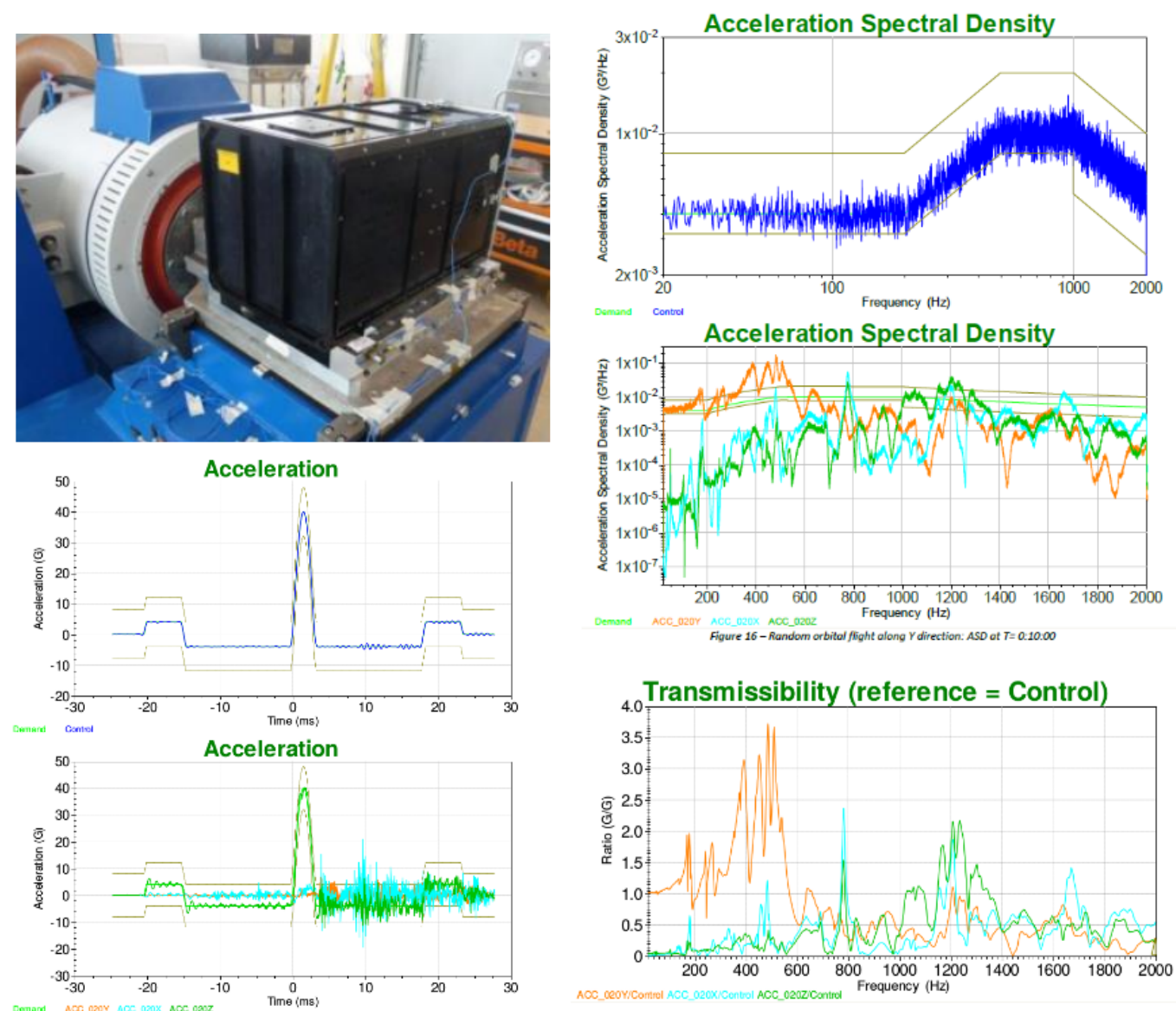

Figure 5: Top Left: Mini-EUSO accomodated on the vibrating plate. Bottom Left: Acceleration response (X, Y and Z) during shock along Y axis. Right: Squared PSD for vibration along Y axis (top) and overall trasmissibility (bottom).

pedestal (low charge accumulation on the anode) from the lower rising slope representing photon reaching the focal surface (see Fig. 6 left). It is possible also to adjust the gain noting that the pedestals, occurring around 150 ADC counts (see Fig. 6 right), are shifted along different channels so the PE production is not uniform over all PDM. Note that the ADC bins are different in the two plot.

Also for ancillary sensors an on-ground calibration was done. We chose $58 \mathrm{~V}$ power supply for the SiPM C13365 array. The DC-DC converter provided by Hamamatsu was programmed through a serial protocol and in Fig. 7, can be seen the range of its linear response to light of the SiPM Hamamatsu matrix. For all optics calibration, sensors are located inside a black box. An integrating sphere focuses UV light emitted by diode onto the sensor's entry window. Two calibrated photodiodes respectively arranged on the sphere and close to sensor collect photons which are converted in light intensity as:

$$
\hat{N}_{p h}=\frac{P_{\text {light }}}{E_{p h}} \simeq\left(2.014 \times 10^{9} \mathrm{ph} / \mathrm{s}\right) \times\left(\frac{P_{\text {light }}}{1 \mathrm{nW}}\right) \times\left(\frac{\lambda}{400 \mathrm{~nm}}\right)
$$



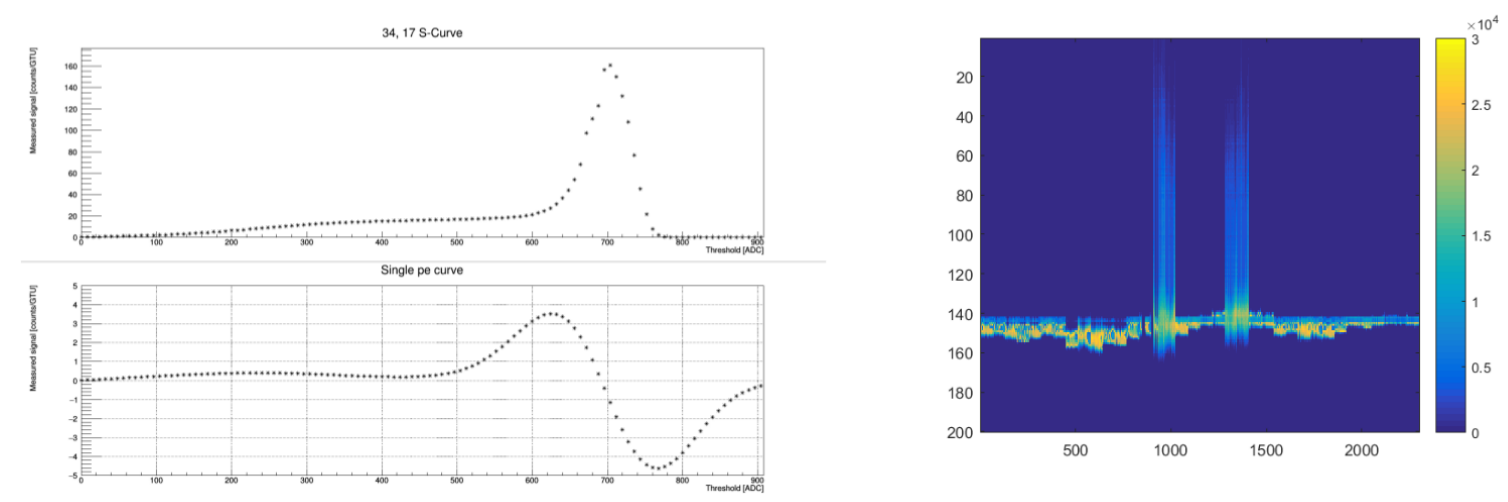

Figure 6: Top Left: Single pixel S-curve. Bottom Left: S-curve derivative. In order to figure out the threshold for the single PE production the derivative must be analyzed. (On x-axis ADC threshold, on y-axis measured counts). Right: Full PDM 2D scan counts. On x-axis are shown the 2304 pixels as a function of ADC threshold (y-axis). Only two ECs are powered and this lead to the two trails in the plot showing triggered pulses counts (showed on the colour bar) also for low ADC levels.

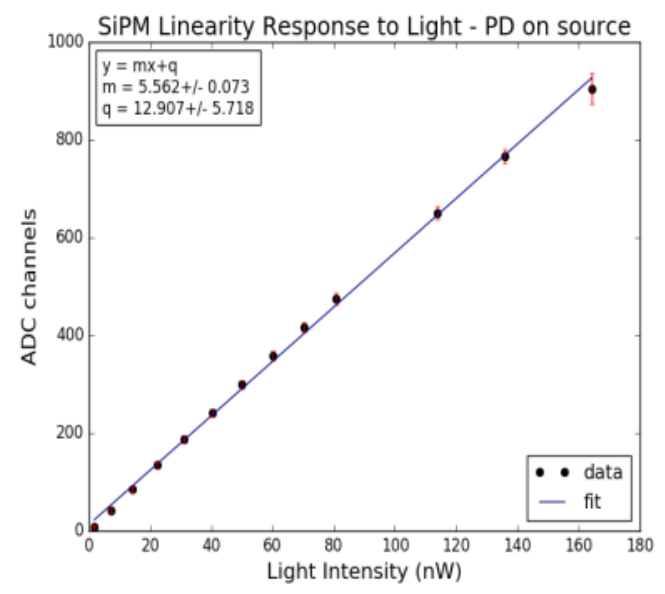

Figure 7: SiPM ADC counts versus light intensity inside a black box.

\section{Conclusion}

Every test was positive and indeed the Mini-EUSO instrument on-board ISS is workin as foreseen. So far, Mini-EUSO have been taking data for more than 40 sessions, more than a thousand of hours and it is still running. This proves the stability and the capability of this kind of detector in space thus laying the foundations for the future missions.

\section{References}

[1] M. Casolino et al.. Mini-EUSO mission to study earth UV emissions on board the ISS. The Astrophysical Journal Supplement Series, 253(2):36, mar 2021. 
[2] M. Casolino, M. Bertaina, A. Belov, T. Ebisuzaki, M. Fukushima, P. Klimov, M. I. Panasyuk, P. Picozza, H. Sagawa, K. Shinozaki, and JEM-EUSO Collaboration. KLYPVE-EUSO: Science and UHECR observational capabilities. International Cosmic Ray Conference, 35:368, January 2017.

[3] A.V. Olintoet al. The POEMMA (probe of extreme multi-messenger astrophysics) observatory. Journal of Cosmology and Astroparticle Physics, 2021(06):007, jun 2021.

[4] S. Blin, P. Barrillon, C. de La Taille, F. Dulucq, P. Gorodetzky, and G. Prevot. SPACIROC3: $100 \mathrm{MHz}$ photon counting ASIC for EUSO-SPB. Nucl. Instrum. Meth., A912:363-367, 2018.

[5] A. Belov, P. A. Klimov, and S. A. Sharakin. The Network Architecture of the Data-processing System for the Photodetector of an Orbital Detector of Ultra-high Energy Cosmic Rays. Instruments and Experimental Techniques, 61:27-33, January 2018. 